\title{
Genetic dissection of pollen competitive ability in maize
}

\author{
M. SARI-GORLA, M. E. PÉ, D. L. MULCAHY,* E. OTTAVIANO \\ Department of Genetics and Microbiology, University of Milano, Milano, Italy and *Department of Botany, University of \\ Massachusetts, Amherst, USA
}

\begin{abstract}
Pollen competition and variability in pollen fitness can produce non-random fertilization with respect to pollen genotypes, and, owing to the large extent of genetic overlap between the gametophytic and sporophytic phases of the life-cycle, can affect the latter. Differences in pollen fitness are due to many factors, of which pollen grain germinability and pollen tube growth rate are the main components. The identification and chromosomal localization of the genes that mainly affect pollen fitness variability were carried out by RFLP analysis, applied to a recombinant inbred population that had been characterized for about 200 restriction loci. Germination ability and pollen tube growth rate were evaluated by means of the pollen mixture technique. Both traits revealed a large variability and high heritability $(0.71 \pm 0.05$ for tube growth rate and $0.77 \pm 0.04$ for grain germinability). Analysis of the association between the expression of the characters and the allelic composition at each of the restriction loci revealed a significant regression on 29 loci in the case of pollen tube growth rate and on 26 in the case of pollen grain germinability. However, considering only uncorrelated loci, in order to avoid false assignments, the minimum number of quantitative trait loci (QTL)s with major effects was five for the tube growth rate and six for grain germinability. The amount of genetic variability of the characters explained by the molecular markers was 0.89 (tube growth rate) and 0.79 (grain germinability), signifying that almost all the genetic variability for these traits is due to QTL located in the chromosomal regions indicated by the analysis. Most of the QTL identified relate to either one trait or the other, which suggests that they are genetically controlled by specific sets of genes.
\end{abstract}

Keywords: competitive ability, maize, pollen, QTL mapping, RFLP analysis.

\section{Introduction}

Pollen competition has been described in many plant species (McKenna \& Mulcahy, 1983; Sarr et al., 1988; Rajora, 1989; Snow \& Spira, 1991), and particularly in maize, where the peculiar floral structure allows the phenomenon to be analysed in detail (Jones, 1928; Mulcahy, 1971; Ottaviano \& Sari-Gorla, 1979; Ottaviano et al., 1988). Variability in pollen fitness can lead to non-random fertilization with respect to pollen genotype, and, because of the special features of the male gametophyte (large population size, haploidy), selection in this phase is expected to be much more effective than selection on the sporophyte. Pollen selection has, in fact, been demonstrated for different traits, such as tolerance to biotic and abiotic stresses, fertility

$\$$ Deceased in June 1991, to whom this paper is dedicated. and plant vigour, and in different species (see Ottaviano \& Mulcahy, 1989 for review). Furthermore gametophytic selection can affect the sporophytic generation and has been suggested as a factor which contributes to the success of the angiosperms (Mulcahy, 1979) and also to the regulation of genetic load (Ottaviano et al., 1988). The biological basis of the phenomenon lies in the large extent of genetic overlap, i.e. the large number of genes that are expressed in both phases of the life cycle (Ottaviano et al., 1991).

Differences in pollen fitness derive from many component factors, including the genetic influence of both the diploid and the haploid genomes (Pfahler, 1975; Ottaviano \& Mulcahy, 1986). The most comprehensive analysis of the genetic control of pollen fitness components was carried out in maize (Ottaviano et al., 1988): grain germinability and tube growth revealed a large amount of variability and high heritability; a signi- 
ficant response to gametophytic selection for tube growth rate and a correlated response for sporophytic traits were detected, proving genetic overlap in the control of the pollen tube growth and the vigour of sporophytic tissues. Moreover, the fertilization ability of a given pollen genotype is modulated by intergametophytic effects (Sari-Gorla \& Rovida, 1980), by environmental effects (Young, 1992) and by interactions with the stylar tissue (Pfahler, 1967; HeslopHarrison, 1975; Sari-Gorla et al., 1976; Herrero \& Arbeloa, 1989).

However, very few data are available concerning the genes that directly control pollen fitness and their specific functions. Pollen competitive ability, and in particular germination rate and tube growth rate, which are its main components, shows the typical features of a quantitative trait (Zamir, 1983; Wendel et al., 1987; Ottaviano et al., 1988; Sarr et al., 1988; Rajora, 1989). This variability can be explained in maize as being the expression of at least two categories of genes (Ottaviano \& Sari-Gorla, 1979): (i) genes specific for pollen development, grain germination and tube growth, and (ii) genes controlling metabolic processes common to pollen and sporophyte. In the first category, in maize, nine different Gametophytic factors $(\mathrm{Ga})$ have been described (Bianchi \& Lorenzoni, 1975). The alleles of these loci confer very different fertilization abilities on gametophytes bearing the dominant or the recessive alleles, favouring the dominant allele when competing in $G a$ styles. It is likely that, in this first category, in addition to the $G a$ factors, various iso-alleles also exist, which have small effects on the pollen phenotype, effects too small to be detected by standard genetic analyses. Their combined action could easily explain at least a part of the observed variability in pollen tube growth rate. Genes of the second category have also to be taken into account, due to the effects of gametophytic selection on sporophytic traits, such as seedling vigour and endosperm development, and the large extent of genetic overlap that has been demonstrated.

The effects on the mating system and population structure differ according to each of these two categories. A multigenic system of $G a$ with minor effects would lead to preferential fertilization by self pollen, best fitted for its own style, thus producing inbreeding; on the other hand, a system of genes controlling metabolic processes should favour allelic combinations adaptive for pollen and sporophytic traits.

The aim of this research is the detection and genetic localization of the genes that mainly affect pollen fitness variability, whether in the first or the second categories. The genetic dissection of pollen competitive ability components (germinability and tube growth rate) was carried out by monitoring the traits by means of molecular markers (RFLP): associations of trait expression and the allelic composition at each marker locus allow the determination of the number of loci mainly involved in the control of the character and the localization of the chromosome regions where they map (Nijenhuis et al., 1987; Osborn et al., 1987; Martin et al., 1989; Keim et al., 1991). The RFLP analysis was applied to a recombinant inbred population, which has been characterized for about 200 restriction loci (Burr et al., 1988).

\section{Materials and methods}

\section{Plant material and experimental design}

We used a family of 45 'recombinant inbreds' (RI), obtained from the cross T232 $\times$ CM37, both standard lines, neither of which carries any dominant $G a$ factors. They were kindly provided by B. Burr, who characterized them for restriction loci: mapping had been done by typing each RI for 200 RFLP loci (see Burr et al., 1988).

The experimental design was a complete randomized block with two replications (days). Five fully grained ears were analysed for each line in each block, for a total of 10 ears per RI.

\section{Competitive ability component evaluation}

Both germinability and pollen tube growth rate were evaluated by means of the pollen mixture technique (Ottaviano et al., 1988): equal amounts of pollen from the line to be tested and from a standard line (W22, genetically marked for coloured aleurone) were used to pollinate 10 plants of the hybrid A632 $\times$ Mo17. W22, A632 and Mo17 are also free of known $G a$ factors. The resulting ears were divided into five segments of equal length; in each segment, with approximately 50-100 kernels per segment, the proportion of ovules fertilized by the two types of pollen was computed on the basis of kernel colour. The proportion of uncoloured kernels in the apical segment indicates the relative germinability of the two pollen types: it is here referred to as pollen grain germinability (PGG). The regression coefficient of the uncoloured kernel proportion on the ear segment, from 1 (Apex) to 5 (Base), is a measure of the pollen tube growth rate (PTGR) relative to that of the coloured standard line. This estimate is based on the peculiar structure of the maize ear: because the length of the silks increases from the apex to the base of the ear, the basal ovules are preferentially fertilized by the faster growing pollen tubes. 


\section{Statistical analysis}

(i) Analysis of pollen trait variability. As the number of kernels per segment was high, but not constant (500-1000), PGG was submitted to a weighted least square analysis of variance, where the weights were the square roots of the reciprocal of the variances (SAS GLM procedure).

For the analysis of PTGR variability and the evaluation of the variances components, a weighted ANOvA was performed by SAS GLM procedure, after a transformation of the original variable (the proportions of uncoloured kernels in the five segments of each ear), according to Ottaviano et al. (1988).

The heritability of both the characters was estimated by standard methods on the basis of the expected mean square values obtained from the ANOVA: the standard errors were calculated according to Baker (1975).

(ii) RFLP analysis. The detection of the chromosome regions where loci controlling the traits are located was based on the association between the expression of the character and the allelic composition at each restriction locus. A regression analysis was performed considering, as dependent variable, the mean value of the measured character (PTGR or PGG) in each line and, as regressor, the allelic composition of each restriction locus, assuming the numerical value of 1,2 or 1.5 according to the presence of two alleles of the first parental line (T232), two alleles of the second (CM37) or alleles of both parental lines (heterozygous locus), respectively. The significance of the regression is interpreted as due to linkage between the restriction locus and a locus controlling competitive ability.

However, in order to avoid false assignments, the correlation matrices between the restriction loci of each chromosome and all the loci giving a significant response were computed. The cumulative contribution of the loci to the character variability was evaluated on the basis of a model including all the significant, noncorrelated, loci.

\section{Results}

The analysis of the traits reveals a large variability for both component characters of pollen competitive ability. The frequency distribution for PTGR and PGG are shown in Fig. 1; the heritability value was $0.71 \pm 0.05$ for PTGR and $0.77 \pm 0.04$ for PGG. This indicates that a large amount of the variability of the traits was due to genetic differences.

The analysis of the association between the expression of the characters and the allelic composition at each of the restriction loci revealed a significant regres-

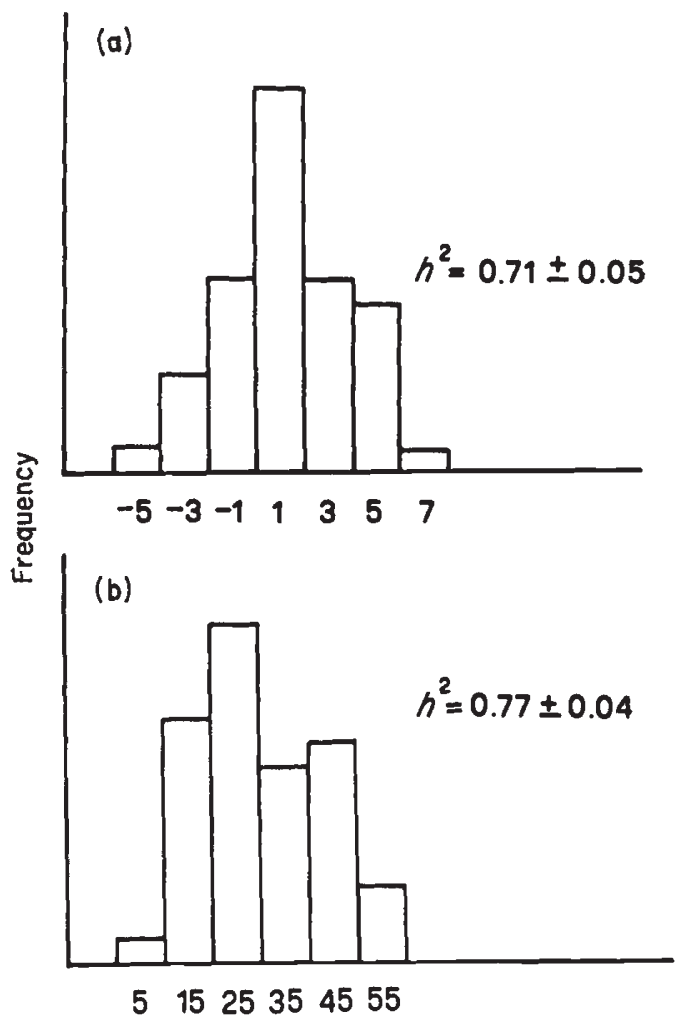

Fig. 1 Recombinant inbred line frequency distribution for pollen tube growth rate (a) and pollen grain germinability (b).

sion on 29 restriction loci in the case of PTGR and on 26 in the case of PGG. The values of the respective $R^{2}$, which measure the proportion of phenotypic variability of the trait explained by allelic substitution at the individual molecular loci, were rather high, ranging from 0.10 to 0.25 . The position on the genetic map of the significant loci for PTGR and for PGG is indicated in Figs 2 and 3, respectively.

The structure of the resulting genetic map reveals that many significant loci are closely linked to each other. This is confirmed by the correlation matrices. Thus it is likely that the significant association found between the character and the different loci of a cluster of marker genes may be due to the presence of only one QTL, localized within the cluster. Accordingly, the significant loci can be grouped into nine separate chromosomal regions for PTGR and in 10 for PGG. The model including one locus per cluster, that with the highest $R^{2}$ value in each cluster, furnishes very high estimates of $R^{2}: 0.71$ and 0.66 , for PTGR and PGG, respectively. These values quantify the amount of phenotypic variation explained by the regressors (restriction loci) included in the model, i.e. due to the association with QTL linked to the molecular markers.

Further information concerning the correlation between marker loci can be obtained from the data 


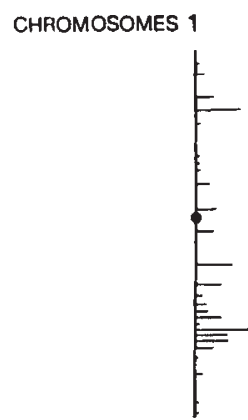

CHROMOSOMES 6

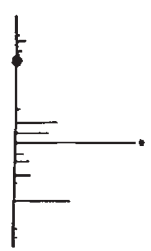

2

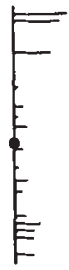

7

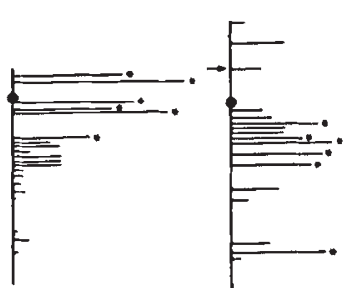

4

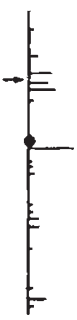

9

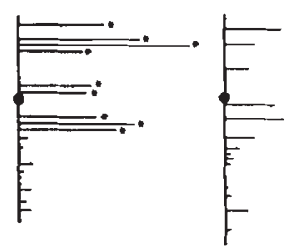

5

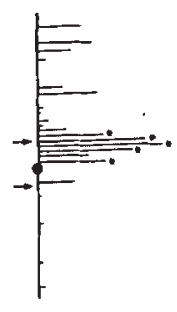

CHROMOSOMES 1

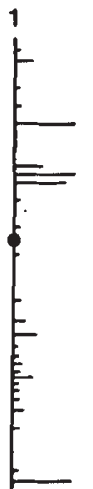

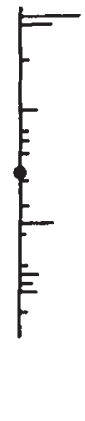

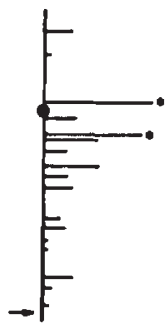

7
8

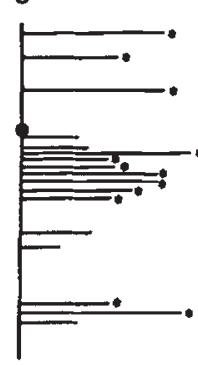

4

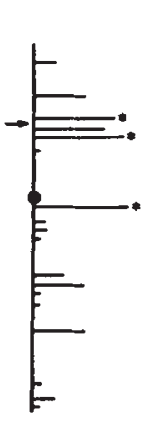

9

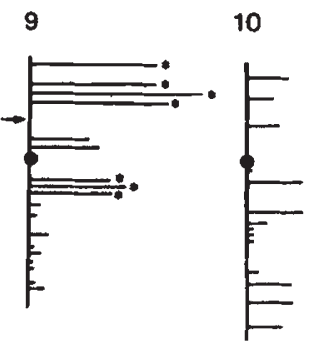

5

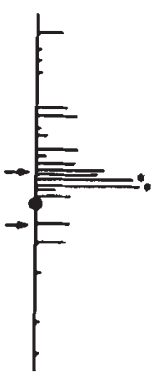

CHROMOSOMES 6
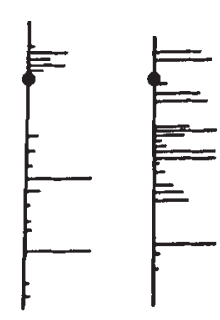

Fig. 2 RFLP analysis of pollen tube growth rate (PTGR). Horizontal bars indicate the degree of correlation between RFLP and PTGR. Approximate localization of known $G a$ factors is indicated by arrows.
Fig. 3 RFLP analysis of pollen grain germinability (PGG). Horizontal bars indicate the degree of correlation between RFLP and PGG. Approximate localization of known $G a$ factors is indicated by arrows. given in Tables 1 and 2; the sign of the regression coefficients indicates which of the parental alleles increases the character; T232 carries the allele 1, CM37 the allele 2; the negative sign indicates that the allelic substitution of 1 with 2 produces a decrease in the value of the character, vice versa a positive value indicates that the allele increasing the character is that of the CM37 parent. The same sign in a sequence of significant restriction loci probably represents a linkage block, where the presence of one or more QTL cannot be distinguished; two blocks of restriction loci characterized by different signs should indicate the presence of different QTL.

Statistical correlation between loci belonging to different chromosomes, theoretically expected to equal zero, can also occur, thus leading to false assignments because sampling variation during inbreeding can produce linkage disequilibrium between restriction loci. This can be detected by analysis of the matrix of correlations including all the significant loci (here not 
Table 1 RFLP loci showing significant effects on the pollen tube growth rate (PTGR)

\begin{tabular}{|c|c|c|c|}
\hline Chromosome & Locus & $R^{2}$ & $b$ \\
\hline \multirow[t]{2}{*}{3} & BNL6.06 & 0.094 & -1.61 \\
\hline & $\operatorname{Pgd} 2$ & 0.136 & -1.45 \\
\hline \multirow[t]{5}{*}{5} & BNL1.380 & 0.091 & -1.61 \\
\hline & BNL10.06 & 0.155 & -2.25 \\
\hline & BNL7.43 & 0.179 & -2.30 \\
\hline & BNL4.36 & 0.137 & -2.11 \\
\hline & Amp3 & 0.102 & -1.37 \\
\hline 6 & NPI223 & 0.188 & 2.31 \\
\hline \multirow[t]{6}{*}{7} & NPI400 & 0.171 & 2.10 \\
\hline & $\mathrm{O} 2$ & 0.265 & 2.71 \\
\hline & BNL15.40 & 0.188 & 1.75 \\
\hline & ZP50 & 0.153 & 1.98 \\
\hline & ZPB36 & 0.240 & 2.46 \\
\hline & BNL15.21 & 0.119 & 1.41 \\
\hline \multirow[t]{6}{*}{8} & NIU1 & 0.139 & 1.37 \\
\hline & BNL1.45 & 0.111 & 1.76 \\
\hline & BNL7.08 & 0.145 & 2.04 \\
\hline & ACT1 & 0.140 & 2.09 \\
\hline & BNL2.369 & 0.120 & 1.92 \\
\hline & NPI268 & 0.145 & 1.82 \\
\hline \multirow[t]{9}{*}{9} & NPI253 & 0.130 & -1.69 \\
\hline & $\mathrm{C} 1$ & 0.185 & -1.69 \\
\hline & Sh1 & 0.256 & -2.57 \\
\hline & $\mathrm{Bz} 1$ & 0.095 & -1.33 \\
\hline & BNL3.06 & 0.111 & -1.76 \\
\hline & Wx1 & 0.105 & -1.75 \\
\hline & Acp 1 & 0.119 & -1.41 \\
\hline & BNL5.04 & 0.175 & -2.24 \\
\hline & BNL7.13 & 0.146 & -2.01 \\
\hline
\end{tabular}

$R^{2}=$ proportion of between-lines variability, $b=$ estimated effects.

reported for brevity): loci whose significant association with the quantitative trait could be the result of their correlation with another marker, strictly linked to a QTL, have been discarded. Considering only uncorrelated chromosomal regions, their number is reduced to 5 and 6 for PTGR and PGG, respectively; the resulting values of $R^{2}$ and 0.63 and 0.60 , are not substantially different from the previous estimates.

As $h^{2}$ estimates the amount of variability for the trait due to genetic differences, and $R^{2}$ measures the amount of variability of the trait due to its dependence on the molecular marker allelic substitution, the ratio between the values of $R^{2}$ and $h^{2}$ evaluates the amount of the genetic variability of the characters explained by the molecular markers. These were $0.89(0.63 / 0.71)$ for PTGR and $0.79(0.60 / 0.77)$ for PGG: this means
Table 2 RFLP loci showing significant effects on pollen grain germinability (PGG)

\begin{tabular}{|c|c|c|c|}
\hline Chromosome & Locus & $R^{2}$ & $b$ \\
\hline \multirow[t]{2}{*}{3} & BNL8.35 & 0.132 & 9.2 \\
\hline & BNL6.06 & 0.116 & 9.60 \\
\hline \multirow[t]{3}{*}{4} & ZPL1A & 0.096 & -8.42 \\
\hline & ZPL1D & 0.107 & -8.86 \\
\hline & ZPL2A & 0.133 & 8.46 \\
\hline \multirow[t]{2}{*}{5} & BNL7.43 & 0.115 & 9.89 \\
\hline & BNI 4.36 & 0.128 & 11.03 \\
\hline \multirow[t]{12}{*}{8} & NPI220 & 0.175 & -10.71 \\
\hline & BNL13.05 & 0.114 & -8.22 \\
\hline & BNL9.11 & 0.117 & -11.90 \\
\hline & NIU1 & 0.208 & -9.07 \\
\hline & BNL9.44L & 0.106 & -9.26 \\
\hline & BNL9.08 & 0.117 & -9.53 \\
\hline & BNL1.45 & 0.164 & -11.55 \\
\hline & BNL7.08 & 0.165 & -11.71 \\
\hline & ACT1 & 0.133 & -10.97 \\
\hline & BNL2.369 & 0.109 & -9.84 \\
\hline & BNL10.24B & 0.109 & -9.14 \\
\hline & NPI268 & 0.199 & -11.51 \\
\hline \multirow[t]{7}{*}{9} & NPI253 & 0.152 & 9.84 \\
\hline & C1 & 0.149 & 10.54 \\
\hline & Sh 1 & 0.212 & 12.58 \\
\hline & Bz1 & 0.161 & 9.33 \\
\hline & Acp 1 & 0.093 & 6.74 \\
\hline & BNL5.04 & 0.108 & 9.49 \\
\hline & BNL7.13 & 0.095 & 8.71 \\
\hline
\end{tabular}

$R^{2}=$ proportion of between-lines variability, $b=$ estimated effects.

that almost all the genetic variability for these traits is due to QTL located in the chromosomal regions indicated by the analysis.

\section{Discussion}

Both PTGR and PGG revealed a high heritability; similar values for the same traits were found when Ottaviano et al. (1988) analysed a synthetic population of maize derived from a gametophytic selection experiment. It is not possible, on the basis of the data reported here, to determine the origin of the genetic variability, i.e. whether it is due to haploid or diploid gene expression. However, previous experiments (Ottaviano et al., 1982, 1988) have shown PGG to be mainly controlled by the sporophyte, while PTGR is, to a large extent, controlled by the gametophyte genome.

The high values estimated for heritability could be explained by the very wide genetic bases from which 
the RI lines were derived. Moreover, particularly in the case of PTGR, the in vivo evaluation minimizes the random effects, thus allowing a very high precision and accuracy of the estimates.

In order to study single components of pollen fitness, the experiment was designed to maintain fixed the effects due to the interaction between pollen and stylar tissues and between pollen genotypes competing in the same style. The pollen-style and pollen-pollen interactions are known to play an important role in the reproductive success of single male gametophytes (Sari-Gorla et al., 1976; Ottaviano \& Sari-Gorla, 1979; Sari-Gorla \& Rovida, 1980; Herrero \& Arbeloa, 1989), and are expected to operate in natural populations.

By considering correlations between linked molecular markers, we obtain the minimum number of QTL with major effects, or, more precisely, the minimum numer of clusters of QTL involved in the trait determination: five for PTGR and six for PGG. However, the actual number of single QTL controlling the traits is likely to have been underestimated. The high number of single molecular markers significantly associated with the traits and their chromosomal position suggest that the QTL involved should be more than five or six. For example, on chromosome 8, for PGG, at least three regions, located near the centromere and at the extremities of the left and right arms, can be clearly detected; they have been considered as a single region, due to the observed correlation but it is reasonable to interpret that they represent at least three distinct QTL effects.

Although, as stated earlier, the classic dominant $G a$ factors are not present in this material, the possibility exists that weakly expressed alleles could be present. Even though it is not possible to establish a precise relationship between the map position of $G a$ factors and the map position of the molecular markers, the chromosomal regions where they are located could be correlated. For example, the cluster of significant markers on chromosome 5 is flanked by two $G a$ loci $\left(G a_{2}\right.$ and $\left.G a_{10}\right)$. On the other hand, $G a_{8}$ on chromosome 9 (Fig. 2) is located in a region between two clusters of significant markers. $G a_{1}$ (Fig. 3) is located close to two significant markers. Moreover, of the nine gametophytic factors detected in maize, only five have been located on chromosomes. However, the effects of allelic substitutions at the $G a$ loci are expected to produce a greater difference in competitive ability between the two pollen types. With regard to the well-characterized gametophytic factors, the proportion of ovules sired by $G a$ pollen tubes in competition with $g a$ on $G a$ silks is about $90-100$ per cent (Nelson, 1952). Thus, if $\mathrm{Ga}$ loci are involved in the determination of the varia- bility in our population, the presence of iso-alleles with minor effects at those loci has to be postulated.

Data obtained by Ottaviano et al. (1983) on the effect of pollen-style interaction on the maize breeding system, suggest the presence of genes demonstrating, in quantitative terms, the same effects as the classical $G a$ factors, and allelic differences with small effects that could easily form a polygenic system. Accordingly, the data reported here indicate the involvement of many genes with minor effects in the determination of the characters: the genetic variability seems to be mainly attributable to segregating QTL.

Most of the QTL delineated are different for the two traits, indicating a genetic control by phase-specific sets of genes. This could be because PGG seems to be mainly supported by gene products translated on mRNA already present in the mature grain, and by reserve material present in the mature grain, and by reserve material provided by the paternal plant; whereas PTGR is sustained by newly activated metabolic processes and depends on interactions with the stylar tissue. In fact, although their specific functions are unknown, many pollen-specific genes have been identified, and some of them isolated (Stinson et al., 1987; Mascarenhas, 1990): it is possible that they control the more important pollen functions, such as the building of the pollen tube wall and cell membrane formation, or the induction in female tissue of nutrient release.

Some of the chromosomal regions involved in the control of the traits are common to both PTGR and PGG. The data reported in Table 1 and 2 show that in these regions, on chromosome 5, 8 and 9, the regression coefficients computed for the two traits have opposite signs; although, in general, it is reasonable to think that the 'same region' does not necessarily mean the 'same QTL'. This observation agrees with the negative correlation that has been observed between the two traits $(r=-0.6)$. A similar phenomenon, an inverse relationship between PGG and PTGR and a curvilinear shape of the growth trend, has already been found in maize (Sari-Gorla et al., 1976; Ottaviano et al., 1988; Landi et al., 1989), suggesting a different pattern of growth during the different phases of pollen function: a high efficiency during germination and the early stage of growth, followed by a phase of lesser vigour, is frequently observed, whereas genotypes with a low growth speed in the initial stage grow faster in the subsequent phases.

As pollen-competitive ability was measured by means of the pollen mixture technique, the data could be interpreted as being due to differences in pollen grain size: larger pollen grains are less frequent in the pollen mixture but may have a greater tube growth rate, 
while smaller pollen grains reveal higher values in the first segment but not during subsequent tube growth. Although a positive relationship between pollen diameter and tube growth rate has been reported only in in vitro conditions (Kumar \& Sakar, 1980), a correlation between pollen size and vigour could be indirectly responsible for a high tube growth rate. However, the significant positive correlation observed between in vivo PGG (measured as proportion of kernels sired in the first segment) and the in vitro pollen germination percentage of the same lines (data not reported), indicates that the indirect evaluation of the trait in vivo is not biased by differences in pollen diameter between the two lines.

\section{Acknowledgements}

The authors are grateful to B. Burr for providing the recombinant inbred lines and information concerning their RFLP typing. The skilful technical assistance of Marzio Villa is greatly appreciated. The research was supported by the National Research Council of Italy, Special Project RAISA, Sub-project 2.

\section{References}

BAKER, W. A. 1975. Manual of Quantitative Genetics. Washington University Press, Pullman.

BIANCHI. A. AND LORENZONI, C. 1975. Gametophytic factors in Zea mays. In: Mulcahy, D. L. (ed.) Gamete Competition in Plants and Animals. North Holland, Amsterdam, pp. 257-263.

BURR, B., BURR, F. A., THOMSON, K. H., ALBERTSON, C. M. AND STUBER, C. W. 1988. Gene mapping with recombinant inbreds in maize. Genetics, 118, 519-526.

HERRERO, M. AND ARBELOA, A. 1989. Influence of the pistil on pollen tube kinetics in peach (Prunus persica). Am. J. Bot., 76, 1441-1447.

HESLOP-HARRISON, J. 1975. Male gametophyte selection and the pollen stigma-interaction. In: Mulcahy, D. L. (ed.) Gamete Competition in Plants and Animals, NorthHolland, Amsterdam, pp. 177-190.

JONES, D. F. 1928. Selective Fertilization. University of Chicago Press, Chicago.

KeIM, P., DIERS, B. W., OLSEN, T. C. AND SHOEMAKeR, R. C. 1990. RFLP mapping in soybean: association between marker loci and variation in quantitative traits. Genetics, 126, 735-742.

KUMAR, D. AND SARKAR, K. R. 1980. Correlation between pollen diameter and rate of pollen tube growth in maize (Zea mays L). Indian J. Exp. Biol., 18; 1242-1244.

LANDI, P., FRASCAROLI, E., TUBEROSA, R. AND CONTI, S. 1989. Comparison between responses to gametophytic and sporophytic recurrent selection in maize (Zea mays $\mathrm{L})$. Theor. Appl. Genet., 77, 761-767.

MCKENNA, M. AND MULCAHY, D. L. 1983. Ecological aspects of gametophytic competition in Dianthus chinensis. In:
Mulcahy, D. L. and Ottaviano, E. (eds) Pollen: Biology and Implications in Plant Breeding, Elsevier, New York, pp. 414-424.

MARTIN, B., NIJENHUIS, J., KING, G. AND SCHAEFER, A. 1989. Restriction fragment length polymorphism associated with water use efficiency in tomato. Science, 243, 1725-1728.

MASCARENHAS, J. P. 1990. Gene activity during pollen development. Ann. Rev. Plant. Physiol. Plant. Mol. Biol., 41, 317-338.

MULCAHY, D. L. 1971. A correlation between gametophytic and sporophytic characteristics in Zea mays L. Science, 171, 1155-1156.

mulCAHY, D. L. 1979. The rise of the Angiosperms: a genecological factor. Science, 206, 20-23.

NELSON, O. 1952. Non reciprocal cross sterility in maize. Genetics, 37, 101-124.

NIJENHUIS, J., HELENTJARIS, T., SLOCUM, M., RUGGERO, B, AND SCHAEFER, A. 1987. Restriction fragment length polymorphism analysis of loci associated with insect resistance in tomato. Crop. Sci., 27, 797-803.

OSBORN, T. C., ALEXANDER, D. C. AND FOBES, J. s. 1987. Identification of restriction fragment length polymorphism linked to genes controlling soluble solids content in tomato fruit. Theor. Appl. Genet., 73, 350-356.

otTaviano, E. AND mulCahy, D. L. 1986. Gametophytic selection as a factor of crop plant evolution. In: Barigozzi, C. (ed.) The Origin and Domestication of Cultivated Plants. Elsevier, Amsterdam, pp. 101-120.

otTaviano, E. AND mulcaHy, D. L. 1989. Genetics of angiosperm pollen. Adv. Genet., 26, 1-64.

OTTAVIANO, E., PE, M. E. AND BINELLI. G. 1991. Genetic manipulation of male gametophytic generation in higher plants. In: Biswas, B. B. and Harris, J. R. (eds) Subcellular Biochemistry 17, Plant Genetic Engineering, Plenum Press, New York, pp. 107-142.

OTTAVIANO, E. AND SARI-GORLA, M. 1979. Genetic variability of male gametophyte in maize. Pollen genotype and pollenstyle interaction. In: Israeli-Italian Joint Meeting. Genetics and Breeding in. Crop Plants. Monographs in Genetic Agraria IV Rome, pp. 89-106.

OTTAVIANO, E., SARI-GORLA, M. AND ARENARI, I, 1983. Male gametophyte competitive ability in maize. Selection and implications with regard to the breeding system. In: Mulcahy, D. L. and Ottaviano, E. (eds) Pollen: Biology and Implications for Plant Breeding, Elsevier, New York, pp. 367-373. otTAVIANO, E., SARI-GORLA, M. AND PE, M. E. 1982. Male gametophytic selection in maize. Theor. Appl. Genet., 63, 249-254.

OTTAVIANO, E., SARI-GORLA, M., PÉ, M. E. AND FROVA, C, 1991. Molecular markers (RFLP and HSPs) for the genetic dissection of thermotolerance in maize. Theor. Appl. Genet., 81, 713-719.

OtTAVIano, E., SARi-GORLA, M. AND Villa, M. 1988. Pollen competitive ability in maize: within population variability and response to selection. Theor. Appl. Genet., 76, 601-608.

PATERSON, A. H., DAMON, S., HEWITT, J. D., ZAMIR, D., RABINOWITCH, H. D., LINCOLN, S. E., LANDER, E. S. AND TANKSLEY, S. D. 1991. Mendelian factors underlying quantitative traits in tomato: comparison across species, generations and environments. Genetics, 127, 181-197. 
PFAHLER, P. L. 1967. Fertilization ability of maize pollen grains. II Pollen genotype, female sporophyte and pollen storage interaction. Genetics, 57, 513-521.

PFAHLER, P. L. 1975. Factors affecting male transmission in maize (Zea mays L) In: Mulcahy, D. L. (ed.) Gamete Competition in Plants and Animals. North Holland, Amsterdam, pp. 115-124.

RAJORA, O. P. 1989. Pollen competition among Populus deltoides Marsh., $P$. nigra L. and $P$. maximowiczii Henry in fertilizing $P$. deltoides ovules and siring its seed crop. Sex. Plant Reprod., 2, 90-96.

SARI-GORLA, M., BELliNTANI, R. AND OTTAVIANo, E. 1976. Competitive ability of maize pollen. Interaction between genotypes of pollen and stylar tissues. Maydica, 21, 77-88.

SARI-GORLA, M. AND ROVIDA, E. 1980. Competitive ability of maize pollen. Intergametophytic effects. Theor. Appl. Genet., 57, 37-41.

SARR, A., SANDMEIER, M. AND PERNES, J. 1988. Gametophytic competition in pearl millet, Pennisetum typhoides (Stapfa and Hubb). Genome, 30, 924-928.

SNOW, A. AND SPIRA, T. P. 1991. Pollen vigour and the potential for sexual selection in plants. Nature, 352, 796-797.

STINSON, J. R., EISENBERG, A. J., WILLING, R. P., PÉ, M. E., HANSON, D. D. AND MASCARENHAS, J. P. 1987. Genes expressed in the male gametophyte of flowering plants and their isolation. Plant Physiol., 83, 442-447.

WENDEL, J. F., EDWARDS, M. D. AND STUBER, C. W. 1987. Evidence for multilocus genetic control of preferential fertilization in maize. Heredity, 58, 297-301.

YOUNG, H. 1992. Environmental effects on pollen characters and paternity. In: Ottaviano, E., Mulcahy, D. L. and SariGorla, M. (eds) Angiosperm Pollen and Ovules. Basic and Applied Aspects. Springer-Verlag, Berlin (in press).

ZAMIR, D. 1983. Pollen gene expression and selection: applications in plant breeding. In: Tanksley, S. D. and Orton, J. J. (eds) Isozymes in Plant Genetics and Breeding. Elsevier, Amsterdam, pp. 313-330. 